\title{
artigo
}

Lehmkuhl, K., Cantos, C. A. R. de Luca, Paulleto, P., Hallal, A. L. C., Bastos, R. C., Cisneros, O., Massignan, C., Cantos, G. de Luca.

Covid-19 e os desafios para o ensino superior: uma revisão de escopo

\section{Covid-19 e os desafios para o ensino superior: uma revisão de escopo}

\author{
Covid-19 and the challenges for higher education: a scoping review \\ Covid-19 y los desafíos para la educación superior: una revisión de alcance
}

\begin{abstract}
RESUMO
Objetivo: Identificar as estratégias e medidas adotadas pelas instituições de ensino superior (IES) a fim de manter as atividades de ensino e aprendizagem durante a pandemia de COVID-19. Método: Foi realizada uma revisão de escopo, onde 5 bases de dados foram consultadas. Foram incluídos estudos que apresentassem medidas preventivas e estratégias para manter as atividades educacionais em instituições de ensino superior durante a pandemia do COVID-19. Resultado: Foram incluídos 16 estudos. As medidas descritas pela maioria dos estudos foram relacionadas a mudanças no calendário acadêmico, uso de tecnologia, aulas online e plataformas de telemedicina. Conclusão: 0 ensino superior, foi profundamente afetado pelo cenário da COVID-19, mas também contou com alta tecnologia para manter as atividades educacionais e, ao mesmo tempo, equilibrar sua missão de proteger os estudantes e professores.
\end{abstract}

DESCRITORES: COVID-19; Educação superior; Educação à Distância

\section{ABSTRACT}

Objective: The present scoping review aims at identifying the strategies and measures adopted by higher education institutions, to maintain teaching and learning activities during the COVID-19 pandemic. Method: A scope review was carried out, where 5 databases were consulted. Studies that presented preventive measures and strategies to maintain educational activities in higher education institutions during the COVID-19 pandemic were included. Result: 16 studies were included. The measures described by most studies were related to making changes in the academic calendar, use of technology, online classes, and telemedicine platforms. Conclusion: Higher education was deeply affected by the COVID-19 scenario, but it also relied on high technology to maintain educational activities and, at the same time, balance its mission to protect students and teachers.

DESCRIPTORS: COVID-19; Education, Higher; Education, Distance

\section{RESUMEN}

Objetivo: Identificar las estrategias y medidas adoptadas por las instituciones de educación superior, para mantener las actividades de enseñanza y aprendizaje durante la pandemia de COVID-19. Método: Se realizó una revisión de alcance, donde se consultaron 5 bases de datos. Se incluyeron estudios que presentaron medidas preventivas y estrategias para mantener las actividades educativas en las instituciones de educación superior durante la pandemia de COVID-19. Resultado: se incluyeron 16 estudios. Las medidas descritas por la mayoría de los estudios estuvieron relacionadas con hacer cambios en el calendario académico, uso de tecnología, clases en línea y plataformas de telemedicina. Conclusión: La educación superior se vio profundamente afectada por el escenario COVID-19, pero también se apoyó en la alta tecnología para mantener las actividades educativas y, al mismo tiempo, equilibrar su misión de proteger a estudiantes y docentes.

DESCRIPTORES: COVID-19. Educación Superior; Educación a Distancia

RECEBIDO EM: 31/08/2021 APROVADO: 01/11/2021

\author{
Karyn Lehmkuhl \\ Bibliotecária, UFSC \\ ORCID: 0000-0002-1041-5491
}

Cleunisse Aparecida Rauen De Luca Canto

Doutoranda do Programa de Pós-Graduação em Engenharia e Gestão do Conhecimento, UFSC ORCID: 0000-0003-0679-8247 


\title{
Patrícia Pauletto
}

Doutoranda do Programa de Pós-graduação em Odontologia, UFSC

ORCID: 0000-0002-1762-7059

\section{Ana Luiza Curi Hallal}

Prof. a Dep. de Saúde Pública, UFSC

ORCID: 0000-0003-4761-0001

\author{
Rogerio Cid Bastos \\ Pró-reitor de Extensão, UFSC \\ ORCID: 0000-0002-3775-1516
}

\section{Odile Cisneros}

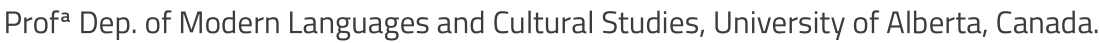
ORCID: 0000-0001-5673-2727

\section{Carla Massignan}

Prof. a Dep. de Odontologia, UnB;

ORCID: 0000-0001-8033-055X

\section{Graziela De Luca Canto}

Prof. ${ }^{a}$ Dep. de Odontologia e Coord. do Centro de Pesquisas Baseadas em Evidências, UFSC;

ORCID: 0000-0002-7986-8317

\section{INTRODUÇÃO}

A COVID-19 obrigou os governos de diferentes países a tomar medidas para restringir a concentração de pessoas. As instituições educacionais em todos os níveis fecharam em 188 países, afetando mais de $91 \%$ da população estudantil global ${ }^{1}$.

Com a suspensão das aulas presenciais, a solução foi migrar o ensino e suas atividades inerentes para o formato virtual. Os protocolos para a transição de aulas presenciais para aulas online tornaram-se uma tendência mundial². Esse cenário constitui-se em um grande desafio para as instituições, assim como para os docentes. Estes se depararam com a necessidade de mudar suas metodologias de ensino e estabelecer novos parâmetros para garantir o processo de aprendizagem ${ }^{3}$.

Muitas inovações já vinham acontecendo no ensino, mas de forma gradual e sem serem mandatórios. Porém, o contexto da pandemia acelerou a adoção de inovações pedagógicas. Assim, em um curto espaço de tempo foi imperativa a adaptação das metodologias para a migração para o ambiente virtual ${ }^{4}$. Mesmo contando com a tecnolo-
Muitas inovações já

vinham acontecendo

no ensino, mas de

forma gradual e sem

serem mandatórios.

Porém, o contexto

da pandemia

acelerou a adoção

de inovações

pedagógicas. gia como aliada, ficou evidente um sistema educacional enraizado em práticas, por vezes ultrapassadas, pautado basicamente em uma única forma de fazer acontecer's.

Para a educação em saúde essa realidade apresentou-se especialmente complicada ${ }^{6}$ Afinal, é uma área do conhecimento na qual os currículos precisam oferecer muitas horas de atividades práticas e com contato direto com pacientes. Uma grande preocupação é como oferecer essas atividades sem expor alunos, professores, funcionários e pacientes ao novo coronavírus. A educação médica teve que se preparar para esse cenário $^{6}$, integrando a tecnologia da informação com metodologias baseadas em problemas para manter o atendimento clínico e garantir a segurança ${ }^{7}$.

Assim, existem inúmeras dúvidas sobre a transição da aprendizagem presencial para a aprendizagem on-line no ensino superior. O que leva ao objetivo desta revisão de escopo o qual pretende responder a seguinte pergunta de pesquisa: Quais são as medidas e estratégias preventivas nas Instituições de Ensino Superior para manter as atividades de ensino e aprendizagem durante a pandemia COVID-19 na educação em saúde? 


\section{artigo}

Lehmkuhl, K., Cantos, C. A. R. de Luca, Paulleto, P., Hallal, A. L. C., Bastos, R. C., Cisneros, O., Massignan, C., Cantos, G. de Luca.

Covid-19 e os desafios para o ensino superior: uma revisão de escopo

\section{MÉTODO}

Foi realizado uma revisão de escopo seguindo o guia de relato PRISMA-ScR8 Um protocolo de pesquisa foi desenvolvido e está disponível no Open Science Framework (OSF) sob o DOI 10.17605/OSF IO/KBDSF, link https://osf.io/37a6q/. Os apêndices 1, 2 e 3 também podem ser visualizados no OSF em https://osf.io/ avbhp/.

Quanto a questão de pesquisa a ser respondida é: Quais são as medidas e estratégias preventivas nas IES para manter as atividades de ensino e aprendizagem durante a pandemia COVID-19 na educação em saúde? Com relação aos critérios de seleção, foram incluídos estudos que apresentassem qualquer tipo de medidas preventivas e estratégias para manter as atividades educacionais em IES, de qualquer país, enquanto as atividades presenciais estivessem restritas ou impedidas de acontecer devido à pandemia do COVID-19. Foram excluídos estudos sobre creches, escolas e pré-escolas. Não foram considerados comentários, cartas, editoriais, resumos de conferências, relatos de casos, revisões sistemáticas, e outros artigos de revisão e informações pessoais. $\mathrm{Da}$ mesma forma, não foram considerados estudos indisponíveis em texto completo, ou artigos em outros idiomas que não o inglês, espanhol ou português. Dentre os estudos sobre o COVID-19 em universidades e faculdades, também foram excluídos aqueles não relacionados ao ensino.

A estratégia de busca foi desenvolvida e executada por um bibliotecário e adaptada para a busca nas seguintes bases de dados: Embase, LILACS, MEDLINE/PubMed, Scopus e Web of Science. As pesquisas foram realizadas em 14 de maio de 2020 . Os resultados não foram filtrados por data, recuperando todos os dados disponíveis nessas bases de dados desde o início da pandemia de COVID-19. As estratégias de busca estão disponíveis no apêndice em https:// osf.io/avbhp/.

A seleção dos estudos foi realizada em duas etapas por dois revisores (KL e CR). $\mathrm{Na}$ fase um, ambos os revisores leram títulos e resumos de forma independente e aplicaram os critérios de elegibilidade. $\mathrm{Na}$ fase dois, os mesmos dois revisores (KL e CR) realizaram a leitura do texto completo novamente aplicando os critérios de eleição. Em ambas as fases, todas as informações recuperadas foram cruzadas pelo terceiro revisor (PP). A seleção final foi baseada no texto completo.

Quanto a coleta e mapeamento dos dados, mais uma vez dois revisores (KL e CR) trabalharam de forma independente reunindo os dados em uma planilha. O próximo passo foi o cruzamento das informações recuperadas com o terceiro revisor (PP), o qual também resolveu eventuais desacordos. Posteriormente, foi gerada uma tabela com os dados coletados, a qual inclui as características dos estudos (autor, ano e país), o tipo de estudo, as características do ambiente/contexto (universidade, residência ou pós-graduação), as principais medidas preventivas e as conclusões. A síntese dos resultados foi realizada de forma narrativa. Sobre as medidas preventivas, entendeu-se como todas as ações ou atitudes adotadas para evitar o contágio da doença e suas consequências, por exemplo, uso de máscara ou distanciamento social. Quanto a estratégias preventivas, considerou-se técnicas ou procedimentos que foram adotados para prevenir o contágio da doença e suas consequências, como as aulas on-line. A síntese dos resultados foi realizada em formato narrativo e os estudos agrupados quanto a área da IES, sendo um grupo da área médica e um grupo para as demais áreas.

\section{RESULTADOS}

A busca nas bases de dados retornou 1.822 referências. Após a remoção das duplicatas, com uso de software gerenciador de referências, restaram 1.502 artigos. Após a leitura dos resumos, 70 foram selecionados para a fase 2 , destes 54 não atenderam aos critérios de elegibilidade (os motivos no apêndice 2). Ao final do processo de seleção, 16 artigos atenderam aos critérios de inclusão. Um resumo de suas características descritivas está disponível no quadro 1 .

O processo de seleção é sintetizado no diagrama do apêndice 3 . Dentre os estudos selecionados, 15 eram artigos de opinião e
01 era estudo observacional. Os estudos relacionados à educação em saúde $\mathrm{e}^{9,10,14,15,17,18,20,21}$ compartilharam preocupações sobre como manter as atividades acadêmicas durante a pandemia, não apenas em relação às aulas, mas principalmente em como proporcionar aos alunos as atividades práticas inerentes à educação médica. As medidas descritas pela maioria dos estudos foram relacionadas a mudanças no calendário acadêmico; estabelecer novas formas de ensino com ferramentas tecnológicas como teleconferência, aulas online, atividades de estudo virtuais; $\mathrm{e}$ o uso de plataformas de telemedicina para atendimento clínico ou ensino. Quando a atividade presencial era impossível de substituir, como para os programas de treinamento de residência, as atividades foram reestruturadas para criar o ambiente mais seguro para residentes e funcionários. Soled et al. ${ }^{21}$ descreveu uma iniciativa criada por estudantes de medicina para envolvê-los na força de trabalho e nos esforços durante a pandemia. $\mathrm{O}$ único artigo sobre educação odontológica $^{13}$, assim como os demais, forneceu orientações a professores e gestores para garantir as atividades educacionais $\mathrm{du}$ rante a pandemia.

Os estudos relacionados à educação superior em geral ${ }^{4,11,12,16,19,20,23}$ também propuseram medidas emergenciais para assegurar as atividades educacionais. Além disso, discutiu-se as consequências da aprendizagem à distância para o futuro do ensino superior e como as inovações pedagógicas continuarão após a pandemia4. A maioria dos estudos concorda que o treinamento é necessário para professores e todas as partes interessadas envolvidas com a educação online e apresentam boas práticas para enfrentar a crise atual e as futuras ${ }^{12,15,16,22}$. Daniel ${ }^{11}$ entende que é imperativo que os alunos tenham igualdade de condiçóes e acesso aos materiais e tecnologia. Gestores acadêmicos e autoridades governamentais não devem aproveitar esse estado de emergência para fazer grandes mudanças no cenário educacional sem um debate adequado ${ }^{19}$

\section{DISCUSSÃO}

Esta revisão de escopo teve como objeti- 


\section{Quadro 1 - Características dos estudos incluídos: autoria, local, tipo de estudo, contexto, medidas e conclusões.}

\section{AUTORIA, ANO, LOCAL E TIPO DE ESTUDO}

Ashokka et al.9, 2020

Singapura

Artigo de opinião

Chick et al.10, 2020

Estados Unidos

Artigo de opinião

Moreno-Correa4, 2020

Colômbia

Artigo de opinião

Daniel11, 2020

Canadá

Artigo de opinião

\section{MEDIDAS E CONCLUSÕES}

Preparação de centros médicos acadêmicos durante a pandemia para cursos de graduação e pós-graduação, residência e atividades de pesquisa.

Manutenção da educação em residência cirúrgica a pandemia.
Estabelecer uma equipe de resposta; adiamento das avaliações; reformulação do calendário acadêmico; estabelecer um processo de comunicação coordenado; estabelecer medidas de segurança e prevenção; incentivo ao relato de sintomas, facilitando a detecção precoce, o isolamento e o manejo.

Recomendações para manter a segurança de alunos, educadores e pacientes; Proposição de soluções inovadoras: implantação de sala de aula virtual; questões práticas on-line; conferências acadêmicas por teleconferências; clínicas de telessaúde com envolvimento de residentes; uso facilitado de vídeos cirúrgicos.

Formação de professores em novas ferramentas educacionais, metodologias de ensino e aprendizagem; professor como tutor que acompanha e orienta o aluno mantendo a motivação do processo de ensino e aprendizagem; mudan-

Conscientização da importância da inovação educacional e pedagógica tendo em vista a situação imposta pela pandemia e as mudanças na educação esperadas no futuro.
Orientação para instrutores, gestores institucionais e governantes para gerenciar as consequências educacionais desta crise pandêmica.
Garantir que os estudantes levem os materiais necessários para estudar; finalizar testes e relatórios; preparação e treinamento de pessoal; aprendizagem assíncrona com videoaulas, uso de recursos educacionais abertos.

Conectar-se com as pessoas e estabelecer confiança mútua; distribuição de liderança em toda a organização; comunicar-se de forma clara e frequente com todas as partes interessadas.
Melhores práticas de liderança para gestores acadêmicos no enfrentamento da crise criada pela pandemia. ça para sala de aula invertida; desenvolvimento de guias de aprendizagem com vídeos explicativos; adaptação de vivência, reflexão e atividades práticas para o ambiente virtual; uso de tecnologias de informação e comunicação para acompanhamento e avaliação pedagógica a fim de cumprir os objetivos educacionais; presença de um monitor acadêmico que para acompanhar os alunos.
Fernandez e Shaw12, 2020

Estados Unidos Artigo de opinião 


\section{artigo}

Lehmkuhl, K., Cantos, C. A. R. de Luca, Paulleto, P., Hallal, A. L. C., Bastos, R. C., Cisneros, O., Massignan, C., Cantos, G. de Luca.

Covid-19 e os desafios para o ensino superior: uma revisão de escopo

Iyer, Aziz e Ojcius13, 2020 Recomendações para educadores e gestores Estados Unidos Artigo de Opinião

Kohan et al.14, 2020

Estados Unidos

Artigo de Opinião

Longhurst et al.15, 2020

Reino Unido

Estudo observacional

Majowicz16, 2020

Canadá

Artigo de opinião

Menon et al.17, 2020

Estados Unidos

Artigo de opinião para garantir a continuidade da educação odontológica durante o surto de COVID-19.
Fluxo de trabalho de cuidados clínicos, supervisão clínica, satisfação no trabalho e orientação para bolsistas durante a pandemia.

Pontos fortes, fracos, oportunidades, ameaças, na adaptação do ensino de anatômica ao contexto online.

Planejamento para professores e universidades em relação ao contexto COVID-19. Responsabilidade institucional pela educação médica, exposição do aluno e garantias de ensino.
Recomendar teletrabalho; estabelecer políticas e protocolos claros; considerações especiais a docentes e funcionários do sexo feminino com filhos pequenos; realizar reuniões on-line para priorizar metas, reorganizar os materiais do curso e obter treinamento em ensino remoto; tranquilizar o corpo docente quanto à sua capacidade de adaptação a esta emergência; oferecer aconselhamento psicológico aos estudantes para lidar com a situação; comunicação aberta e informações precisas disponíveis para alunos, professores, funcionários e pacientes.

Melhores evidências sobre o uso de plataformas remotas e virtuais em cuidados clínicos; adaptação às alterações na gestão clínica; fornecer orientação sobre o impacto na pós-graduação.

Fortes: novas aulas online, aprimoramento de tecnologias e recursos. Fracos: limitações de tempo, falta de sessões práticas, problemas de avaliação. Oportunidades: colaboração acadêmica, trabalho remoto, incorporação de aprendizado hỉbrido no currículo. Ameaças: diminuição da relação professor/aluno e redução do envolvimento dos alunos.

Fatores macroambientais com potencial para influenciar o

funcionamento da academia no âmbito do COVID-19; análise de cenários futuros para testar as decisões ou tomada de decisões.

Assegurar segurança dos estudantes promovendo ensino funções essenciais e não educacionais; manter os estudantes de medicina formados "sob reserva" para servir quando forem necessários.

Mukhopadhyay et al.18

2020

Estados Unidos

Artigo de opinião
Ensino de patologia e tecnologia para educação e aprendizagem remotas.
Curadoria de recursos on-line que podem ser usados para aprendizagem virtual em patologia; reuniões virtuais. 
Murphy19, 2020

Canadá

Artigo de opinião

Análise dos protocolos de eLearning emergenciais adotados por universidades durante o surto COVID-19.

Reestruturação de emergência de um programa de residência em cirurgia geral durante a pandemia de COVID-19.

Estados Unidos

Artigo de opinião

Soled et al.21, 2020

Estados Unidos

Artigo de opinião

Wendelboe et al.22, 2020

Estados Unidos

Artigo de opinião

Varalakshmi e Arunacha-

lam23, 2020

India

Artigo de opinião

Criação da equipe de resposta formada por estudantes de medicina no enfrentamento da COVID-19. Preparação da universidade para lidar com a
crise do COVID-19.

Iniciativas para manter alunos ativos e vinculados às atividades durante o distanciamento social e quarentenas.
Uso da teoria de securitização da Escola de Copenhagen para análise dos protocolos; a normalização do e-Learning de emergência significaria a normalização de uma forma de educação que perpetua as desigualdades estruturais.

Promoção do bem-estar da força de trabalho, limitando o número de residentes com contato direto com o paciente e criação de equipes que trabalham isoladas umas das outras.

Desenvolvimento de uma estrutura organizacional par otimizaria a capacidade de mobilizar de forma eficiente os pares interessados na resposta COVID-19; servir como um elo entre administração e os gestores do hospital.

Criação de um exercício para auxiliar as universidades em sua preparação para a pandemia.

Cursos online abertos massivos, vídeos gravados, streaming de vídeo, avaliações usando quiz, tarefas colaborativas, compartilhamento de recursos online.

Fonte: Elaborado pelos autores.

vo identificar medidas preventivas adotadas pela IES para manter as atividades educacionais durante a crise provocada pela nova pandemia do vírus corona. A maioria dos estudos recuperados relacionava-se à educação em saúde, mas foram incluídos estudos sobre o ensino superior em geral. Outra observação importante é que, como o corpus do estudo é composto em sua maioria por artigos de opinião, as informações coletadas refletem principalmente experiências empíricas e perspectivas pessoais.

A maioria dos estudos apresentou as ferramentas online como os principais recursos para manter as atividades de aprendizagem e pesquisa. Essa mudança levou as instituições de saúde a interromper práticas educacionais tradicionais e apontou para a urgência na implementação de estratégias alternativas de educação e avaliação ${ }^{7,15,24}$.

Embora a tecnologia tenha se apresentado como tábua de salvação, certas atividades foram severamente prejudicadas, por exemplo, as que requerem contato direto com o paciente. Devido às restrições re- comendadas pelos Centros de Controle e Prevenção de Doenças e outras organizações ${ }^{10}$, programas de residência médica tiveram que adaptar-se à estratégia de rotação para minimizar a exposição dos residentes ao vírus, mantendo a distância necessária para prevenir o contágio ${ }^{20,25}$. Como essa crise provocada pelo novo coronavírus é uma situação inédita, os estudos revelam que existe um consenso entre educadores e lideranças acadêmicas para permitir reajustes curriculares, mudanças nos calendários acadêmicos e maior flexibilidade nas metodologias de avaliação. Assim, a metodologia de ensino, o papel do professor e sua relação com os alunos foram impactados ${ }^{3}$. O uso de tecnologia para apoiar a educação requer criatividade e adaptabilidade em resposta aos contextos específicos e mutáveis em que é usado ${ }^{26}$.

Espera-se que essa mudança para atividades educacionais online afete profundamente a educação de agora em diante. Educadores e líderes não devem se esquecer de propor debates adequados sobre como usar a tecnologia na educação de uma forma que não crie desigualdades ${ }^{19}$. $\mathrm{O}$ uso da tecnologia irá reforçar a visão instrumental capitalista da educação ou promoverá o crescimento humano holístico? Os gestores educacionais devem pensar cuidadosamente sobre as decisões que estão tomando e como elas pavimentam o caminho da educação no futuro ${ }^{27}$.

A educação presencial retornará, mas os arranjos especiais colocados em prática durante este período de pandemia e suas consequências deixarão um rastro duradouro $^{11}$. Dito isso, as instituições de ensino superior devem investir em recursos tecnológicos, mas principalmente em seus recursos humanos. As ferramentas online para educação certamente estarão cada vez mais presentes, por isso é imprescindível que todos os profissionais envolvidos com a educação saibam como utilizá-las, quando seriam mais adequadas e quando não seriam. Em suma, falar sobre o impacto do novo coronavírus no setor educacional vai além de uma (re) organização estrutu- 


\section{artigo}

Lehmkuhl, K., Cantos, C. A. R. de Luca, Paulleto, P., Hallal, A. L. C., Bastos, R. C., Cisneros, O., Massignan, C., Cantos, G. de Luca.

Covid-19 e os desafios para o ensino superior: uma revisão de escopo

ral dos cursos. Implica mudança de atitude para gestores, professores e alunos para que (re) formulem as práticas de ensino. É necessário que as práticas sejam inovadoras, proporcionando ao aluno criticidade, reflexão, interação, diálogo e vínculo. Esses são elementos essenciais para a transformação, não apenas a transmissão de conhecimento.

Quanto às limitações percebidas neste estudo, a primeira delas é o pequeno número de publicações que responderam à questão de pesquisa. Ademais, em sua maioria foram artigos de opinião, o que pela urgência do tema, era algo esperado. Outro ponto é que não foram localizados estudos relatando se as medidas tomadas de fato funcionam.

\section{CONCLUSÃO}

Os desafios enfrentados pelas instituições de ensino superior durante a pandemia do COVID-19 estavam principalmente relacionados a encontrar maneiras de forne- cer experiências de ensino e aprendizagem de boa qualidade e ao mesmo tempo lidar com o distanciamento social. $\mathrm{O}$ ensino superior, em especial o ensino da saúde, foi profundamente afetado por esse cenário, mas também contou com alta tecnologia para manter as atividades educacionais $\mathrm{e}$ ao mesmo tempo, equilibrar sua missão de proteger os estudantes e professores.

\section{REFERÊNCIA}

1. UNESCO. Education: from disruption to recovery [Internet]. 2020 [cited 2020 Aug 18]. Available from: https://en.unesco.org/covid19/ educationresponse

2. Bezerra IMP. State of the art of nursing education and the challenges to use remote technologies in the time of Corona Virus Pandemic. J Hum Growth Dev [Internet]. 2020 Apr 14;30(1):141-7. Available from: http://revistas.marilia.unesp.br/index.php/jhgd/article/view/10087

3. Kenski VM. Tecnologias e ensino presencial e a distância. 6th ed. São Paulo: Papirus; 2008.

4. Moreno-Correa SM. La innovación educativa en los tiempos de Coronavirus. Salut Sci Spiritus [Internet]. 2020;6(1):14-26. Available from: https://revistas.javerianacali.edu.co/index.php/salutemscientiaspiritus/article/view/2290/2863

5. Santos AT dos, Almeida JMC de, Rolim ILTP, Passos HM, Coutinho NPS, Sardinha AH de L. Educação a distância através do ensino remoto durante a COVID-19 em um mestrado acadêmico em enfermagem: relato de experiência. Rev Saúde Coletiva [Internet]. 2021;11(COVID):6973-7. Available from: http://revistas.mpmcomunicacao.com.br/index.php/ saudecoletiva/article/view/1755/2046

6. Newman NA, Lattouf OM. Coalition for medical education-A call to action: A proposition to adapt clinical medical education to meet the needs of students and other healthcare learners during COVID 19. J Card Surg [Internet]. 2020 Jun 30;35(6):1174-5. Available from: https:// onlinelibrary.wiley.com/doi/abs/10.1111/jocs.14590

7. Lall S, Singh N. CoVid-19: Unmasking the new face of Education. Int J Res Pharm Sci [Internet]. 2020 Apr 17;11(SPL1):48-53. Available from: https://pharmascope.org/ijrps/article/view/2122

8. Tricco AC, Lillie $E$, Zarin W, O'Brien KK, Colquhoun $H$, Levac $D$, et al. PRISMA Extension for Scoping Reviews (PRISMA-ScR): Checklist and Explanation. Ann Intern Med [Internet]. 2018 Oct 2;169(7):467. Available from: http://annals.org/article.aspx?doi=10.7326/M18-0850

9. Ashokka B, Ong SY, Tay KH, Loh NHW, Gee CF, Samarasekera DD. Coordinated responses of academic medical centres to pandemics: Sustaining medical education during COVID-19. Med Teach [Internet]. 2020 Jul 2;42(7):762-71. Available from: https://www.tandfonline.com/doi/ full/10.1080/0142159X.2020.1757634

10. Chick RC, Clifton GT, Peace KM, Propper BW, Hale DF, Alseidi AA, et al. Using Technology to Maintain the Education of Residents During the COVID-19 Pandemic. J Surg Educ [Internet]. 2020 Jul;77(4):72932. Available from: https://linkinghub.elsevier.com/retrieve/pii/ S1931720420300842

11. Daniel SJ. Education and the COVID-19 pandemic. Prospects
[Internet]. 2020 Apr 20;(April). Available from: http://link.springer. com/10.1007/s11125-020-09464-3

12. Fernandez AA, Shaw GP. Academic Leadership in a Time of Crisis: The Coronavirus and COVID 19. J Leadersh Stud [Internet]. 2020 May 11;14(1):39-45. Available from: https://onlinelibrary.wiley.com/doi/ abs/10.1002/jls.21684

13. Iyer $P, A z i z K$, Ojcius DM. Impact of COVID-19 on dental education in the United States. J Dent Educ [Internet]. 2020 Jun;84(6):718-22. Available from: http://doi.wiley.com/10.1002/jdd.12163

14. Kohan L, Sobey C, Wahezi S, Brancolini S, Przkora R, Shaparin N et al. Maintaining High-Quality Multidisciplinary Pain Medicine Fellowship Programs: Part II: Innovations in Clinical Care Workflow, Clinical Supervision, Job Satisfaction, and Postgraduation Mentorship for Pain Fellows During the COVID-19 Pandemic. Pain Med [Internet]. 2020 Aug 1;21(8):1718-28. Available from: https://academic.oup.com/painmedicine/article/21/8/1718/5831836

15. Longhurst GJ, Stone DM, Dulohery K, Scully D, Campbell T, Smith CF. Strength, Weakness, Opportunity, Threat (SWOT) Analysis of the Adaptations to Anatomical Education in the United Kingdom and Republic of Ireland in Response to the Covid 19 Pandemic. Anat Sci Educ [Internet]. 2020 May 9;13(3):301-11. Available from: https://onlinelibrary.wiley. com/doi/abs/10.1002/ase.1967

16. Majowicz SE. What might the future bring? COVID-19 planning considerations for faculty and universities. Epidemiol Infect [Internet] 2020 Apr 29;148(May):e92. Available from: https://www.cambridge. org/core/product/identifier/S0950268820000898/type/journal_article

17. Menon A, Klein EJ, Kollars K, Kleinhenz ALW. Medical Students Are Not Essential Workers: Examining Institutional Responsibility During the COVID-19 Pandemic. Acad Med [Internet]. 2020 Aug 28;95(8):1149-51. Available from: https://journals.Iww.com/10.1097/ ACM.0000000000003478

18. Mukhopadhyay S, Booth AL, Calkins SM, Doxtader EE, Fine SW, Gardner JM, et al. Leveraging Technology for Remote Learning in the Era of COVID-19 and Social Distancing. Arch Pathol Lab Med [Internet]. 2020 Sep 1;144(9):1027-36. Available from: http://meridian.allenpress.com/aplm/article/144/9/1027/442318/Leveraging-Technology-for-Remote-Learning-in-the

19. Murphy MPA. COVID-19 and emergency eLearning: Consequences of the securitization of higher education for post-pandemic pedagogy. Contemp Secur Policy [Internet]. 2020 Jul 2:41(3):492-505. Available from: https://www.tandfonline.com/doi/full/10.1080/13523260.2020 


\section{REFERÊNCIA}

.1761749

20. Nassar AH, Zern NK, Mclntyre LK, Lynge D, Smith CA, Petersen $\mathrm{RP}$, et al. Emergency Restructuring of a General Surgery Residency Program During the Coronavirus Disease 2019 Pandemic. JAMA Surg [Internet]. 2020 Jul 1;155(7):624. Available from: https://jamanetwork. com/journals/jamasurgery/fullarticle/2764317

21. Soled D, Goel S, Barry D, Erfani P, Joseph N, Kochis M, et al. Medical Student Mobilization During a Crisis: Lessons From a COVID-19 Medical Student Response Team. Acad Med [Internet]. 2020 Sep 4;95(9):1384-7. Available from: https://journals.Iww.com/10.1097/ ACM. 0000000000003401

22. Wendelboe AM, Miller A, Drevets D, Salinas L, Miller EJ, Jackson $D$, et al. Tabletop exercise to prepare institutions of higher education for an outbreak of COVID-19. J Emerg Manag [Internet]. 2020 Mar 16;18(2):183-4. Available from: https://wmpllc.org/ojs/index.php/jem/ article/view/2725

23. Varalakshmi R, Arunachalam K. COVID 2019: role of faculty members to keep mental activeness of students. Asian J Psychiatr [Internet]. 2020 Jun;51(April):102091. Available from: https://linkinghub.elsevier. com/retrieve/pii/S1876201820302021
24. Liu Y, Jin GF, Wang JM, Xia YK, Shen HB, Wang CQ, et al. [Thoughts on the reform of preventive medicine education in the context of new medicine]. Zhonghua Yu Fang Yi Xue Za Zhi [Internet]. 2020 Jun 6;54(6):593-6. Available from: http://www.ncbi.nlm.nih.gov/ pubmed/32253890

25. Kania K, Abu-Ghname A, Agrawal N, Maricevich RS. Four Strategies for Plastic Surgery Education amid the COVID-19 Pandemic. Plast Reconstr Surg [Internet]. 2020 Aug 5;146(2):252e-253e. Available from: https://journals.Iww.com/10.1097/PRS.0000000000007122

26. Ellaway R, Masters K. AMEE Guide 32: e-Learning in medical education Part 1: Learning, teaching and assessment. Med Teach [Internet]. 2008 Jan 3;30(5):455-73. Available from: http://www.tandfonline. com/doi/full/10.1080/01421590802108331

27. Teräs M, Suoranta J, Teräs H, Curcher M. Post-Covid-19 education and education technology 'Solutionism': a seller's market. Postdigital Sci Educ [Internet]. 2020 Oct 13:2(3):863-78. Available from: http:/ link.springer.com/10.1007/s42438-020-00164-x 